\title{
LOS RETOS DE LA CO-GENERACIÓN EN LA BÚSQUEDA DEL IMPACTO SOCIAL DE LA UNIVERSIDAD: UN CASO DE CONSTRUCCIÓN DE UN ESPACIO DiAlógICo A TrAVÉS DE LA INVESTIGACIÓN ACCIÓN
}

CHALLENGES FOR CO-GENERATION IN THE SEARCH FOR SOCIAL IMPACT OF THE UNIVERSITY: A CASE OF THE CONSTRUCTION OF A DIALOGIC SPACE THROUGH ACTION RESEARCH

\author{
Patricia Canto $^{1^{*}}$, Pablo Costamagna ${ }^{2}$, Andoni Eizagirre $^{3}$ y Miren Larrea ${ }^{1}$ \\ Orkestra-Instituto Vasco de Competitividad, Universidad de Deusto, España ${ }^{1}$ \\ *autora de contacto: pcanto@orkestra.deusto.es \\ Universidad Tecnológica Nacional, FFRA/ Universidad Nacional de Rafaela, Argentina ${ }^{2}$ \\ Mondragon Unibersitatea, España ${ }^{3}$
}

\begin{abstract}
Resumen: Al centro del debate sobre el impacto social de la universidad se encuentra una nueva división del trabajo en la producción de conocimiento que pueda responder a los retos de la sociedad. Esta nueva división del trabajo es más compleja y advierte un giro epistemológico en la forma de organizar la interacción entre conocimiento académico y sociedad. Este artículo plantea cuatro retos identificados a partir de un caso de construcción de un espacio dialógico para la co-generación de conocimiento. El caso, desarrollado a través de la investigación acción, cuestiona el esquema que empaqueta el conocimiento generado en la universidad en forma de artículos, libros, informes o conferencias y que lo comunica de forma lineal y plantea otro basado en el diálogo que permite a los otros actores aportar su propio conocimiento.
\end{abstract}

Palabras clave: universidad, impacto social, investigación acción, comunicación.

\section{Introducción}

El debate sobre el impacto social de la universidad está tomando fuerza en los últimos años. Al centro de este debate se encuentra la capacidad de la universidad de interactuar con otros actores de la sociedad para co-generar conocimiento que contribuya a hacer frente a los retos que afrontan los territorios. Las políticas de las administraciones públicas alimentan también este debate con una apuesta clara por una investigación transdisciplinar orientada a la solución de los grandes retos sociales.

No obstante, para que la universidad pueda cogenerar conocimiento con otros actores de la sociedad necesita replantear su misión de transferencia como una misión de servicio a la sociedad (Tandon et al., 2016). Debe también arrojar una mirada crítica al monopolio que ha mantenido en la producción de conocimiento y a su estructura en silos disciplinares. Asimismo sus investigadores necesitan desarrollar capacidades específicas como la escucha activa y la comunicación en diálogos multidireccionales en tiempo real (Oswald et al., 2017); la gestión del conflicto, las relaciones de poder y saber generar capital social (Karslen \& Larrea, 2015). La comunicación, el lenguaje y la mediación se señalan también como capacidades necesarias para que la universidad juegue un papel de agente de cambio en el territorio (Aranguren et al., 2016; Trencher et al., 2014).

Este artículo profundiza en la discusión anterior e intenta responder a la pregunta de cuáles son los retos de la universidad para co-generar el conocimiento con otros actores de la sociedad. Cuestiona, en concreto, el esquema que empaqueta el conocimiento generado en la universidad en forma de artículos, libros, informes o conferencias y lo comunica de forma lineal a los otros actores 
del territorio, sin que exista un diálogo que permita a estos aportar su propio conocimiento.

El artículo se divide en tres apartados. El primer apartado examina la relación entre la universidad y la sociedad y pone de manifiesto la necesidad de explorar una nueva división del trabajo en la producción de conocimiento que pueda responder a los retos de la sociedad. El segundo apartado presenta el caso dividido en tres secciones. La primera sección plantea el contexto en el que se desarrolla el caso. La segunda sección explica el punto de partida del caso: el borrador de un libro que inicialmente iba a comunicarse bajo el esquema lineal descrito en el párrafo anterior. La tercera sección describe el proceso de construcción de un espacio dialógico para comunicar dicho conocimiento a través de la investigación acción. El último apartado del artículo presenta la discusión del caso a modo de conclusión.

\section{La universidad y su relación con la sociedad}

La concepción de la investigación en la universidad, en nombre de la objetividad ante los problemas, tradicionalmente se ha sostenido por un planteamiento demarcacionista y lineal entre aspectos científicos y sociales de la investigación. Aquella tradición vinculaba la investigación académica a la búsqueda de conocimiento verdadero, fecundo y coherente, regida exclusivamente por valores cognitivos y un conjunto de métodos mediante los cuales se certifica el conocimiento genuino. A esta particularidad se sumaba otra de carácter normativo, según la cual la garantía de la buena ciencia, o de la investigación responsable, es la propia acción del investigador en base al respeto de las normas científicas (Douglas, 2009). Asimismo, el investigador, guiado por la curiosidad y su anhelo por descubrir e interpretar la evidencia interna del mundo real, físico y social, aislaba de sus reglas y normas las consecuencias del uso del conocimiento generado y la satisfacción de las necesidades humanas y el conocimiento beneficioso para la sociedad (Brown \& Guston, 2009).

En este sentido, la universidad y su relación con la sociedad se han regido por una clara división funcional y moral del trabajo (Van den Daele, 1978). Más concretamente, la autoridad epistémica y la organización de la investigación han estado sometidas a una serie de presupuestos que guían y dan sentido a la producción de conocimiento. La perspectiva sociológica clásica señala que "el objetivo institucional de la ciencia es la extensión del conocimiento certificado" (Merton, 1942: 270). Esta peculiaridad de la ciencia se observa sobre un principio que ha reconocido y estimulado la labor del investigador, a saber, la libertad e independencia de la investigación académica con el objetivo de generar el conocimiento por su propio bien. Unido a ello, el reconocimiento estandarizado y profesional se ha establecido en virtud del grado de verosimilitud, el valor científico, la originalidad y la publicidad del conocimiento (Polanyi, 1961). A todo ello precede una visión lineal y positivista de la investigación, que ha beneficiado un modelo de proceder y evaluar caracterizado por la metodología cuantitativa, el modelo acumulativo del conocimiento científico y una lógica con pretensión universal.

Esta imagen de la investigación y su relación con la sociedad, nunca exenta de debate, está sometida a una revisión continuada (Rip, 2012). Al respecto existen consideraciones muy diversas: algunas insisten en la naturaleza compleja de los retos que como sociedad se deben acometer, otras reparan en la contribución económica y social de la universidad, y otras más destacan la ampliación del capital cognitivo a organizaciones ajenas al entramado académico y universitario, o resaltan una imagen diferente de los resultados esperados. En cualquier caso, estos cambios, aunque de forma y grado desiguales, informan de la necesidad de estimular programas y estrategias de colaboración para activar la intensidad, calidad y densidad de la investigación. Dicho de otra manera, aquellas consideraciones dejan entrever un giro epistemológico y social en la forma de comprender y organizar las interacciones entre el conocimiento académico y la vida institucional, comunitaria y empresarial (Bonaccorsi \& Daraio, 2007).

Todo ello viene a demandar políticas de vinculación socialmente más robustas, a la vez que los planteamientos demarcacionistas arriba subrayados se muestran altamente inoperantes (Felt \& Wynne, 2007). A ello se debe que paulatinamente las políticas de investigación ponen mayor énfasis en los problemas y desafíos a resolver, en vez de en las disciplinas y áreas de conocimiento, a la vez que una mayor sensibilidad contextual acentúa el carácter distribuido y colectivo del conocimiento (Kuhlmann \& Rip, 2018). De esta manera, la suposición tradicional 
sobre el descubrimiento experto como revelación de verdades objetivas, está siendo desplazada por una perspectiva según la cual los distintos valores, conocimientos y expectativas se integren en los procesos de investigación e innovación y los resultados se alineen mejor con las distintas visiones, necesidades y preferencias sociales (Eizagirre, 2017).

Esto, de alguna manera, cuestiona que la peculiaridad de la investigación en la universidad sea la consideración de que el propio conocimiento es el principal producto y propósito de las investigaciones. No obstante, la emergencia de una nueva modalidad de organizar, producir y validar el conocimiento en contextos de aplicación, que transciende los roles prestablecidos, no está reñida con la calidad y excelencia científica (Ziman, 1998). En este mismo sentido, un interés creciente por la resolución de problemas y la investigación orientada por retos no comporta necesariamente el rechazo de la idea de que la ciencia sea una estrategia epistémica de un valor considerable y, de sus características sociales.

Por el contrario, el reconocimiento paulatino de la naturaleza distribuida de la experteza y la colaboración de organizaciones distintas, todas ellas portadoras de conocimientos, valores e intereses, favorece la adaptación a contextos, ambientes y usuarios diferenciados, al mostrar una mayor variedad, robustez y sensibilidad contextual (Callon et al., 2009). Estos cambios vienen a señalar una nueva división del trabajo, más compleja e interactiva, que advierte un régimen de investigación distribuido, en el que interactúan y colaboran distintos profesionales y públicos de la sociedad civil, como respuesta a las necesidades y aspiraciones sociales.

Ello requiere, sin embargo, una singular perspectiva sobre la naturaleza, el sentido y los propósitos de la universidad. Por una parte, somete la autoridad epistémica y la organización de la investigación a la cooperación con otros actores y propósitos extra-académicos. Por otra parte, aboga por comprender la universidad en términos más relacionales y reivindica el carácter abierto de los procesos de investigación e innovación (Eizagirre et al., 2017).

\section{Etorkizuna Eraikiz como espacio de experimentación del rol co- generativo de la universidad: el caso del espacio de encuentro para facilitadores}

\subsection{Introducción al caso}

El apartado anterior planteaba la necesidad de explorar una nueva división del trabajo, más compleja e interactiva a la hora de generar conocimiento que responda a los problemas de la sociedad. Para ello, se decía, es necesaria la interacción y colaboración entre investigadores universitarios de diferentes disciplinas y entre estos y distintos profesionales y públicos de la sociedad civil.

El caso que se aborda a continuación es un proyecto desarrollado con el objetivo de construir un espacio de encuentro para facilitadores del desarrollo territorial en el contexto de Etorkizuna Eraikiz, programa liderado por la Diputación Foral de Gipuzkoa (DFG). Dicho proyecto se puede interpretar como un proceso experimental de construcción de un espacio para la colaboración no sólo entre investigadores sino entre estos $\mathrm{y}$ distintos profesionales vinculados, en este caso, a la facilitación del desarrollo territorial. Se trata por lo tanto de un experimento sobre el rol interactivo de la universidad con otros actores del territorio. Siguiendo el lenguaje utilizado en la investigación acción, que inspiró la metodología del proceso en el caso, se trata de un experimento sobre el rol cogenerativo de la universidad en su objetivo de generar conocimiento útil para la resolución de los problemas de la sociedad.

El papel co-generativo de la universidad se abordó en el proyecto desde la perspectiva de la comunicación del conocimiento académico, campo que una de las autoras de este artículo estaba abordando en el contexto de su tesis doctoral. El planteamiento de partida era que una universidad que quiere co-generar el conocimiento para que este responda de forma más directa a las necesidades de la sociedad, no puede seguir un esquema de comunicación lineal con la sociedad. En dicho esquema, el conocimiento se genera en la universidad y después se comunica a los actores en formatos como los informes, artículos, libros, seminarios sin que los actores del territorio tengan la opción de dialogar con los investigadores y contribuir a la construcción de dicho 
conocimiento. Desde ahí se planteó un reto a dos de los autores de este artículo, para que experimentaran una forma distinta de comunicar un borrador de libro sobre el que estaban trabajando. Dichos contenidos se habían construido pensando en el formato libro, por lo que respondían al proceso habitual de producción académica. Sin embargo, el trabajo realizado a partir de esos borradores permite hablar de una nueva forma de comunicar el conocimiento académico que avanza en la dirección planteada en el marco teórico rompiendo la tradicional división del trabajo.

\subsection{El punto de partida: el borrador de un libro}

Tal y como se señalaba, el punto de partida del proceso fue un borrador escrito por dos de los autores de este artículo, que iba a comunicarse en forma de libro. El contenido del libro podría, a priori, parecer intrascendente en términos de la reflexión de cómo se comunicó. Sin embargo, de cara a trasladar con más claridad quiénes fueron los actores con los que posteriormente se cogeneró conocimiento, conviene entender cuál era el contenido de dicho borrador. Es por ello que en esta sección se describe el punto de partida del proceso: el borrador que posteriormente se convirtió en un libro de la Serie de Desarrollo Territorial de Deusto con el título Actores Facilitadores del Desarrollo Territorial. Una Aproximación desde la Construcción Social (Costamagna \& Larrea, 2017).

El libro plantea un argumento inicial de que las personas facilitadoras son un elemento relevante para que los procesos de desarrollo territorial funcionen, y sin embargo, su papel se ha conceptualizado muy poco y cuando se ha hecho, se les ha atribuido un papel neutral que no responde a la interpretación que los autores del libro hacen de su naturaleza en la práctica. Por ello, el libro plantea los pasos para reinterpretar esta figura y ayudar al lector a encontrarse a sí mismo como persona facilitadora de procesos de desarrollo territorial.

El primer paso en este camino es entender el origen de esta interpretación de la facilitación, que es doble: el enfoque pedagógico (gestado en América Latina) y la investigación acción para el desarrollo territorial (que tiene sus raíces en la colaboración entre entornos de investigación del País Vasco y Noruega). Estas aproximaciones permiten argumentar que el desarrollo territorial es un proceso complejo y los procesos complejos no se pueden desarrollar en términos de ordeno y mando ni permiten, a través del análisis, "encontrar" las soluciones. Dichas soluciones hay que "construirlas" y ello requiere desarrollar procesos de construcción social. El libro sostiene que en el desarrollo territorial frecuentemente no se han distinguido los problemas complicados (en los que existe una solución que se puede descubrir a través del conocimiento experto) de los complejos (problemas en que no existe una solución a descubrir, sino que hay que construirla de forma contextual). Por ello se ha intentado resolver problemas complejos como complicados, a través de lógicas lineales y decisiones jerárquicas. La figura de la persona facilitadora se plantea, en cambio, como alguien que puede generar las condiciones para que otros actores reflexionen, decidan y actúen para construir las soluciones de forma colaborativa.

Tras plantear este marco, los autores del libro proponen la figura del actor facilitador, que rompe con la imagen de facilitador profesional neutral y propone que los distintos actores del territorio, ya sean de la política, la empresa y la universidad, pueden tener intereses propios y aun así ser legitimados por otros actores para facilitar un proceso. Una vez definida esta figura, el libro aborda el liderazgo del actor facilitador, que se define como un liderazgo relacional. El libro se plantea además la pregunta de si los facilitadores nacen o se hacen $\mathrm{y}$, por lo tanto, si es posible formar a facilitadores. Partiendo de una hipótesis de trabajo de que sí lo es, se plantean una serie de roles de las personas facilitadoras y las capacidades individuales y colectivas que se requieren para que dichos roles se puedan abordar.

El objetivo de los autores del libro es provocar la reflexión del lector sobre su propio papel en el desarrollo territorial viendo si el marco conceptual de la facilitación y sus características ayudan a entender parte de sus experiencias vividas.

Este contenido era apropiado para experimentar una nueva forma de comunicar conocimiento académico no sólo porque los autores del libro estuvieran dispuestos a participar en el proceso, sino también porque el propio contenido está orientado a generar capacidades de facilitación en un contexto de multidisciplinariedad y co-generación. Por lo tanto, era previsible que los potenciales lectores de este libro fueran personas con una actitud a priori favorable a participar en un proceso co-generativo. 
Considerando la participación directa de todos los autores de este artículo en el proceso de investigación acción que construyó el espacio dialógico, se ha optado por utilizar la tercera persona del plural en las siguientes secciones.

\subsection{La construcción, a través de la investigación acción, de un espacio dialógico para comunicar el libro sobre facilitadores}

Para responder a la pregunta de "cómo" comunicar el libro sobre actores facilitadores, desarrollamos un proceso de investigación acción basado en el modelo co-generativo de Greenwood y Levin (2007). La investigación acción se plantea como una estrategia para el cambio que mediante ciclos de reflexión-acción-reflexión genera aprendizajes que resuelven un problema en la práctica y generan nuevo conocimiento relevante en el ámbito académico.

Tal y como señalábamos, en el proceso de investigación acción participamos los cuatro autores de este artículo. Partíamos del objetivo definido en términos de "comunicar el libro de forma co-generativa" pero jugamos diferentes roles. Los autores del libro jugaron el rol de dueños del problema, mientras la autora que en aquel momento realizaba su tesis doctoral, diseñó el proceso a partir de un marco analítico construido como resultado de una revisión de la literatura sobre Investigación e Innovación Responsables (IIRs) y Comunicación para el Desarrollo y el Cambio Social (CDCS). Por su parte el cuarto autor, que desempeñó el rol de amigo crítico, mantuvo un diálogo constante con la doctoranda, ayudándola a reflexionar sobre el desarrollo del proceso y sugiriendo nuevas líneas de discusión. También se integraron, en diferentes momentos, profesionales con conocimiento sobre tecnologías de la información; comunicación y diseño audiovisual y gestión de proyectos. En la última etapa participó también un grupo piloto formado mayoritariamente por doctorandos e investigadores.

En cada uno de los talleres los marcos conceptuales presentados por la doctoranda disparaban la reflexión de los autores del libro a la luz del reto planteado de "cómo" comunicar el conocimiento sobre la figura de los actores facilitadores. A partir de la reflexión los autores tomaban decisiones que se llevaban a la acción. Al finalizar el proceso dichas acciones se habían materializado en un espacio digital dialógico para comunicar el conocimiento sobre la figura de los actores facilitadores. La Tabla 1 presenta un resumen del proceso.

Tabla 1. El proceso de investigación acción: conceptos y decisiones tomadas

\begin{tabular}{|c|l|}
\hline $\begin{array}{c}\text { Concepto/ } \\
\text { fecha del taller }\end{array}$ & \multicolumn{1}{c|}{ Decisiones } \\
\hline $\begin{array}{c}\text { La comunicación } \\
\text { como proceso. } \\
\text { (Marzo 7, 2017) }\end{array}$ & $\begin{array}{l}\text { Diseñar un viaje en dos etapas: } \\
\text {-Un viaje interactivo en el que los viajeros interiorizan los aportes de los autores y } \\
\text { los de los viajeros que han pasado antes que ellos por las diferentes paradas. } \\
\text {-Un lugar de encuentro al final del camino en el que el libro se siga escribiendo. }\end{array}$ \\
\hline $\begin{array}{c}\text { La interacción de } \\
\text { conocimientos } \\
\text { diferentes. } \\
\text { (Abril 12, 2017) }\end{array}$ & $\begin{array}{l}\text {-Diseñar el viaje interactivo con espacios que permitan visualizar que en cada } \\
\text { parada del viaje tiene lugar un diálogo diferido entre los autores y los viajeros sobre } \\
\text { los diferentes capítulos del libro. }\end{array}$ \\
\hline $\begin{array}{c}\text { La inclusión de } \\
\text { perspectivas, } \\
\text { vivencias y } \\
\text { expectativas. } \\
\text { (Mayo 9, 2017) }\end{array}$ & $\begin{array}{l}\text {-Formular preguntas en cada parada que inviten a los viajeros a reflexionar sobre los } \\
\text { capítulos del libro conectando con su propio conocimiento teórico y práctico. }\end{array}$ \\
\hline $\begin{array}{c}\text { Comunidades de } \\
\text { aprendizaje. } \\
\text { (Junio 13, 2017) }\end{array}$ & $\begin{array}{l}\text {-Que el lugar de encuentro al final del camino se construya a partir de un diálogo } \\
\text { emergente entre los viajeros que hayan completado el viaje. } \\
\text {-Que la responsabilidad de mantener vivo el diálogo sea colectiva. }\end{array}$ \\
\hline
\end{tabular}

Fuente: Elaboración propia. 


\subsection{El resultado: un espacio para el encuentro de actores facilitadores}

El resultado tangible del proceso descrito en la sección anterior, es un espacio abierto en la web (https://dgroups.org/groups/perfadt) en que se invita a los participantes a realizar un viaje que desemboca en un ágora o plaza en la que se pueden encontrar con otros viajeros interesados en la facilitación del desarrollo territorial.

Una de las preocupaciones constantes a lo largo de los talleres fue la necesidad de diseñar un proceso dialógico realista y sostenible. Esta reflexión fue clave en el diseño del espacio como un proceso dividido en las dos etapas señaladas anteriormente: el viaje y la plaza.

\section{a. El viaje interactivo}

El viaje interactivo, se plantea como un diálogo diferido que se desarrolla a lo largo de un recorrido con diferentes paradas. En cada una de ellas los viajeros tienen la posibilidad de acceder tanto a los capítulos originales escritos por los autores como a material audiovisual preparado por los autores para acercar dichos capítulos a los viajeros. Pero sobre todo, los viajeros tienen la posibilidad de compartir sus reflexiones sobre los contenidos del libro y leer las contribuciones que han ido dejando los viajeros que han pasado por dichas paradas antes que ellos. Este diálogo diferido es, siguiendo los criterios de realismo y sostenibilidad, la fórmula intermedia entre la ausencia total de diálogo y el diálogo en tiempo real entre autores y lectores del libro. Esta fórmula intermedia se consideró necesaria porque el formato tradicional de comunicación del conocimiento a través de un libro no hubiera dado lugar para desarrollar ningún tipo de diálogo pero, a su vez, la exigencia de mantener un diálogo continuo hubiera resultado insostenible para los investigadores. El concepto de diálogo diferido permitió encontrar un punto intermedio entre ambos extremos.

La Figura 1 muestra una de las paradas del viaje. El espacio de la izquierda captura la voz de los autores del libro a través de textos y material audiovisual. Los vídeos en los que los autores se dirigen directamente a los viajeros complementan los capítulos del libro. El espacio de la derecha captura las reflexiones que al respecto hacen los viajeros.

Figura 1. Un viaje interactivo en el que se desarrolla un diálogo diferido

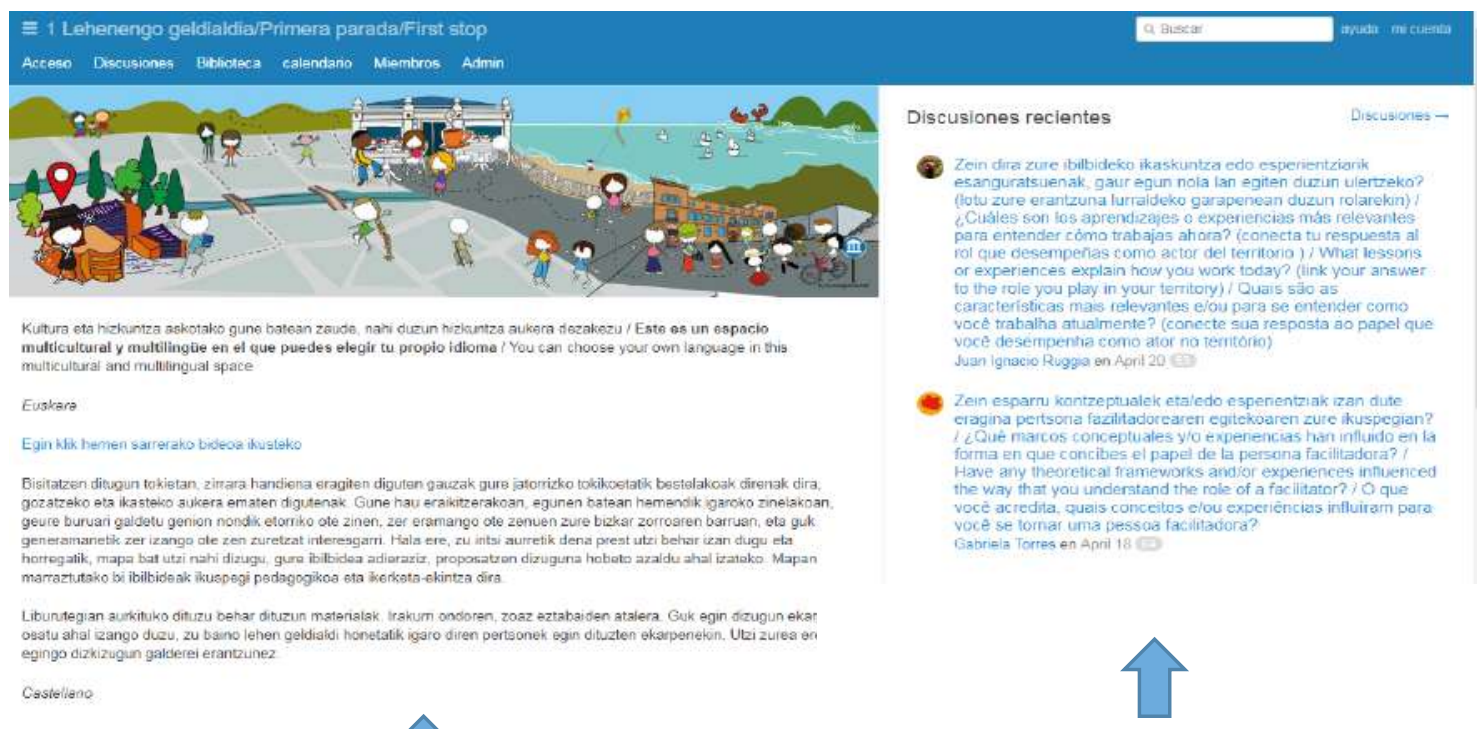

Voz de los viajeros

Voz de los autores

Fuente: https://groups.org/groups/perfadt 
En el momento de escribir este artículo han iniciado el viaje 183 personas localizadas en distintos lugares del mundo: Argentina: 73; País Vasco: 51; Uruguay: 22; Brasil: 9; México: 6; Finlandia: 5; Colombia: 3; Perú: 3 y una persona de cada uno de los siguientes: Alemania, Chile, Cuba, Ecuador, Italia, Kenia; Nigeria, Noruega, Reino Unido, República Dominicana y Vietnam. Entre ellas hay docentes, investigadores y hacedores de políticas públicas y privadas. b. El ágora o plaza como punto de encuentro

Por su parte, la segunda etapa del proceso, menos vinculada a los contenidos directos del libro y más directamente interactiva se definió como un espacio en el que "seguir escribiendo el libro". Se configuró como un ágora, un espacio emergente que exige una implicación más continua a los autores pero en el que la responsabilidad de mantener el diálogo vivo es colectiva. Es decir, el libro se sigue escribiendo, pero ya no únicamente por los autores sino por todas las personas que forman parte del espacio y plantean debates y reflexiones sobre la facilitación del desarrollo territorial. La Figura 2 muestra este espacio emergente.

Figura 2. Un espacio emergente en el que seguir escribiendo el libro

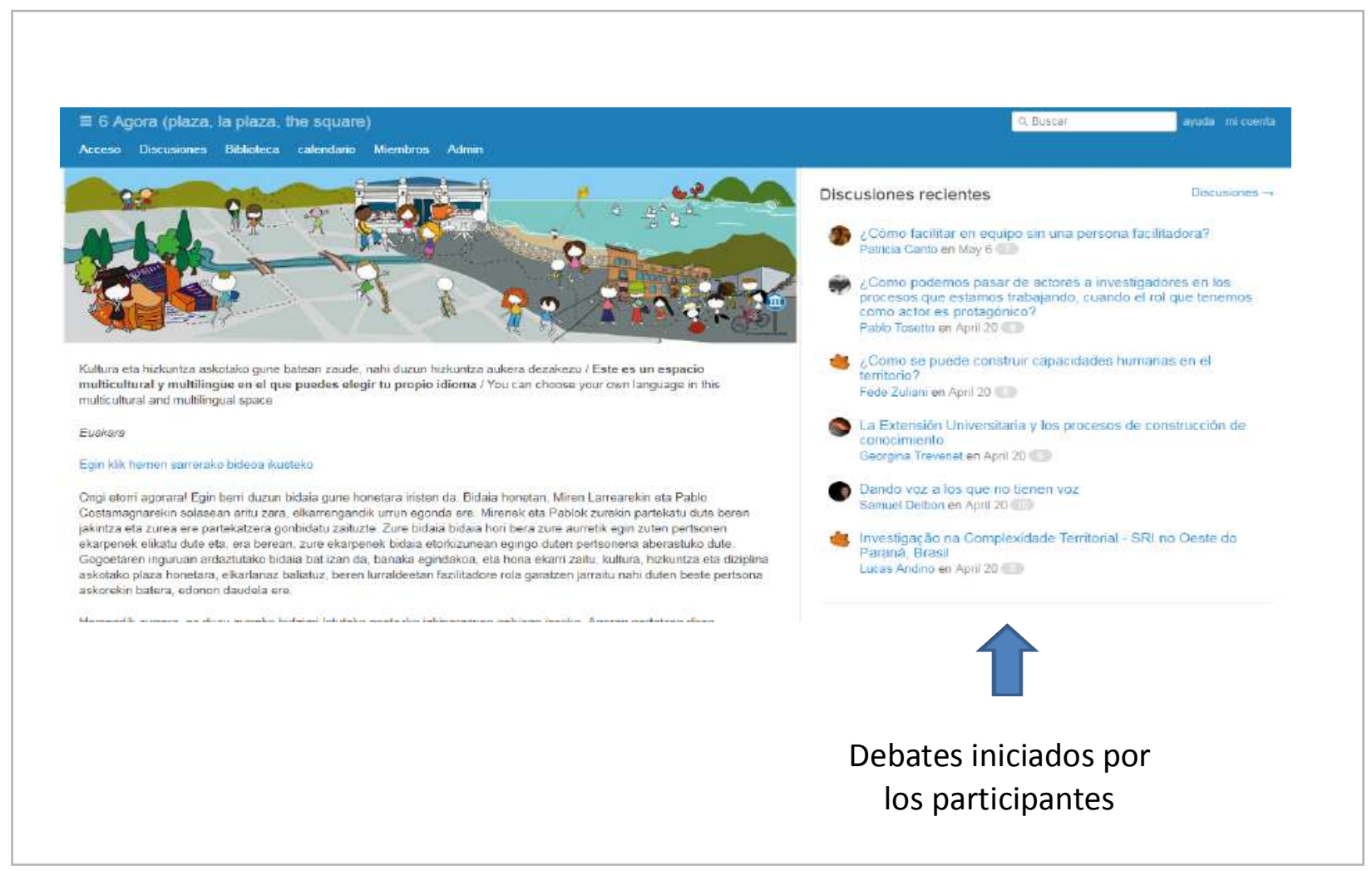

Fuente: https://dgroups.org/groups/perfadt

En el momento de escribir este artículo, de las 183 personas que iniciaron el viaje, 54 se han integrado al ágora y 15 personas han participado en los debates planteados. La distribución de estas personas es Argentina: 31; País Vasco: 10; Uruguay: 6; Finlandia: 3; y una persona de Brasil, Italia, México y Nigeria.
Como ejemplo de una de las discusiones desarrolladas en el ágora, presentamos la siguiente, que conecta con la discusión sobre la invisibilidad de la persona facilitadora planteada en el libro ${ }^{1}$ :

\footnotetext{
${ }^{1}$ Se cuenta con el permiso de todos los autores de los fragmentos incluidos en este artículo para su publicación en el mismo.
} 
"Yo me animo a compartir una experiencia muy reciente vivida en un proyecto de investigación-acción que facilito. En un momento dado, me di cuenta de que se me estaba invisibilizando como facilitadora. Ocurrió cuando una de las policy -makers del proceso, en una presentación para presentar su estrategia para apoyar el desarrollo local, se refirió a las acciones definidas como resultado del proceso de investigación-acción pero no mencionaba el espacio de reflexión-acción que comparte con nosotros, los investigadores $[\ldots] "$.

Espacio de Facilitadores, 18 de noviembre de 2017

Un fragmento de la discusión que siguió a esta intervención es:

"Creo que parte de la no valoración del rol de los facilitadores en los procesos de desarrollo territorial, se debe a que muchas de las personas facilitadoras creíamos en la importancia de la invisibilidad de ese rol. Hoy considero lo contrario".

Espacio de Facilitadores, 21 de noviembre de 2017

Este debate generó un total de 24 intervenciones en las cuáles diferentes actores del territorio (investigadores $\mathrm{y}$ otros) entablaron un diálogo sobre este aspecto que se aborda en el libro pero que en el diálogo se reformula a partir de las experiencias de los diferentes actores.

Entendemos que con estos procesos se materializa un proceso de co-generación de conocimiento vinculado al libro, pero que trasciende los contenidos presentados en el mismo. Por ello interpretamos que este modo de comunicar el libro responde a los nuevos modos co-generativos que se le plantean como reto a la universidad y que pueden abrir caminos para procesos de construcción de conocimiento cogenerativos y orientados a la resolución de problemas concretos. Se modifica la división del trabajo, hay un enfoque colectivo donde vale el conocimiento de los actores.

Una de las características de este proceso ha sido que en el punto de partida se contaba ya con unos contenidos elaborados por los investigadores. El planteamiento por lo tanto no sustituye el esfuerzo inicial de conceptualización y teorización de los investigadores por un espacio en que el conocimiento se construye de forma dialógica. Lo que se plantea es la complementariedad de ambas aproximaciones en un marco en el que hay momentos en que los investigadores trabajan por su cuenta y momentos en que interactúan con sus "lectores" en nuevos procesos de construcción de conocimiento.

\section{Discusión del caso: conclusiones}

Tras haber compartido el marco teórico y el caso práctico, pasamos a continuación a explicitar las conexiones entre los mismos para poder construir las conclusiones del artículo. Considerando que estamos ante un debate que toma fuerza, pero para el que la universidad no siempre tiene respuestas, planteamos la discusión no en términos de recomendaciones, sino en torno a cuatro retos:

a) Crear conocimiento socialmente relevante;

b) Superar la división de funciones en la creación de conocimiento: la universidad lo crea y la sociedad lo asimila;

c) La legitimación del conocimiento cogenerado;

d) El cambio de cultura dentro de la universidad para poder co-generar.

Un primer reto que identificábamos para la universidad en el primer apartado es el de generar conocimiento socialmente relevante. Para ello es necesario trascender los planteamientos epistemológicos, metodológicos y teóricos que organizan la producción del conocimiento alrededor de silos disciplinares ya que la complejidad de los retos de la sociedad demanda miradas configuradas desde la interacción entre diferentes disciplinas.

La multidisciplinariedad se ha materializado en el proyecto de dos formas distintas. En primer lugar, el diseño del espacio interactivo de comunicación del libro sobre actores facilitadores es el resultado de una mirada multidisciplinar (economía, comunicación, filosofía, tecnologías de la información y diseño audiovisual) a un problema concreto: la necesidad de que la universidad aporte su conocimiento de una manera que contribuya al cambio en el territorio. Por otra parte, se trata de un espacio diseñado para experimentar con la interacción entre diferentes disciplinas y entre diferentes actores académicos y extra-académicos. 
Una de las personas que terminó el viaje compartió que lo que más le había gustado fue:

“...el poder conocer la experiencia y opinión de otros actores. Sobre todo, el debate interno que me ha surgido al discrepar o entender de otra manera lo expuesto y el poder trabajarlo para ser capaz de construir una visión conjunta."

Cita tomada del cuestionario que responden los viajeros que han terminado el viaje interactivo (11 de enero de 2018).

Un segundo reto es que no es suficiente con salir de la lógica de silos disciplinares, también es necesario superar la división de funciones por la que frecuentemente se sobreentiende que la universidad produce el conocimiento $\mathrm{y}$ posteriormente la sociedad lo recibe y lo aplica. La estrategia interactiva de comunicación del conocimiento sobre actores facilitadores intenta superar esa división creando un espacio en el que "el libro se siga escribiendo" pero en donde la responsabilidad de escribirlo ya no es solo de los autores sino de todos los que conforman dicho espacio. Esta transición a nuevas formas de producción de conocimiento tiene la difícil tarea de aunar los criterios de calidad científica con la relevancia social y la sensibilidad contextual.

Ello requiere un cambio en la universidad, puesto que se amplían y descentralizan los ámbitos dotados de conocimiento, de manera que debe saber desplegar nuevas capacidades vinculadas al reconocimiento mutuo, la colaboración y el aprendizaje cooperativo. Pero no sólo la universidad se ve ante la necesidad de cambiar. Este tipo de proceso también demanda variaciones en otros ámbitos de la sociedad en los que los actores territoriales deben pasar de receptores pasivos a co-generadores de conocimiento, con los consiguientes miedos, falta de práctica y tiempos dedicados a la reflexión. Uno de los participantes del espacio de facilitadores lo expresaba de la siguiente manera:

"El esfuerzo está por comprender que es un espacio de aprendizaje colectivo, en donde todas las opiniones generan aportes...fácil decirlo, pero un poco más complejo llevarlo a cabo"...

Espacio de Facilitadores, 6 de abril de 2018
Lo anterior nos lleva al tercer reto, que se planteaba en el plano teórico en el primer apartado: ¿Cómo legitimar en la academia el nuevo conocimiento co-generado entre ciencia y sociedad? El carácter co-generativo mejora las posibilidades de que el conocimiento generado ayude a resolver los problemas al integrar en el proceso el conocimiento de los actores que mejor conocen dichos problemas. Pero al no ser un conocimiento producido bajo los supuestos imperantes sobre la producción científica, el conocimiento co-generado plantea dificultades a la hora de integrarlo de nuevo en el debate académico. Es por ello que, para que un modo de generación de conocimiento colaborativo y abierto no suponga una penalización en la trayectoria de los investigadores, es importante construir mecanismos de legitimación de este tipo de conocimiento en la academia.

A modo de ejemplo, una investigación que integre en su diseño distintas perspectivas, incluya diferentes actores y diversifique las fuentes del conocimiento, reflexione activamente en aras a integrar las consideraciones socio-económicas y sea flexible para cambiar la trayectoria y su orientación como respuesta a los aprendizajes, los valores y las necesidades, apela a una profunda revisión de los supuestos epistemológicos y metodológicos tradicionales sobre la naturaleza y el sentido de la investigación. A ello se suman otros obstáculos, como son aquellos referidos a los tiempos y a los propósitos de la actividad investigadora, a la estructura organizativa y funcional de las universidades, o a los modelos y criterios que regulan e incentivan los distintos programas de evaluación de la calidad y acreditación. En relación con esto último, las estructuras evaluativas sobre acreditación nacional que regulan el sistema de promoción profesional y sus instituciones, que controlan e incentivan la investigación, las publicaciones y la carrera académica en las universidades, están claramente pensadas para un modelo muy singular de universidad que plantea dificultades para integrar propuestas como la presentada en este artículo. La pregunta queda abierta: ¿Cómo se legitima el conocimiento co-generado en el registro que propone este artículo?

Relacionado con los anteriores, un cuarto y último reto es el cambio de cultura requerido en la universidad para poder integrar con naturalidad los modelos co-generativos. ¿Cómo confronta hoy en día la universidad la llamada a la co-generación? 
¿Cómo se posicionan los investigadores individuales ante la facilitación de procesos de cogeneración de conocimiento entre actores diferentes? Esto requiere un profundo cambio en las rutinas y conductas que superan la decisión individual del investigador. En el fondo, se trata de repensar las dimensiones que afectan al proceso y el propósito de la investigación. En el párrafo anterior se han señalado algunas de las dificultades y resistencias, muchas de ellas de un carácter organizativo y estructural. En cualquier caso, hay que tejer también mimbres para un cambio cultural dentro de la universidad y a corto plazo, en el contexto concreto que se ha analizado en este artículo, dicho cambio puede llegar de la mano de cursos de doctorado y de formación de investigadores en los que se planteen este tipo de procesos. El espacio construido para el encuentro de facilitadores puede servir este objetivo.

En ese sentido, creemos necesario poner en valor la experimentación. Los autores de este artículo hemos tenido, gracias a un programa de la administración pública (Etorkizuna Eraikiz), la oportunidad de experimentar con un espacio en el que el conocimiento académico se comparte de forma dialógica. Esta oportunidad ha creado las condiciones para generar capacidades en la propia universidad y en otros actores del territorio en el marco de la única gobernanza posible en este camino: aquella en la que los diferentes actores, investigadores y no investigadores, colaboran en la construcción del conocimiento que ayude a superar los retos del territorio.

\section{Agradecimientos}

Los autores agradecen a Rakel Vázquez, Maite Reizabal e Idoia Egaña la contribución de su conocimiento en la construcción del espacio de encuentro. Agradecen a la Diputación Foral de Gipuzkoa el apoyo recibido para el desarrollo del proyecto "Universidad y gobernanza colaborativa el despliegue de las capacidades de facilitación".

\section{Referencias}

Aranguren, M. J., Franco, S., Horta, R. and Silveira, L. (2016). Retos y aprendizajes de institutos de investigación transformadora. Journal of Technology Management and Innovation, 11(1), 69-79. DOI: 10.4067/S071827242016000100010

Bonaccorsi, A. and Daraio, C. (2007). Universities as Strategic Knowledge Creation. Specialization and Performance in Europe. Cheltenham: Edward Elgar Publishing. DOI: 10.4337/9781847206848

Brown, M. and Guston, D. (2009). Science, democracy, and the right to research. Science and Engineering Ethics, 15(3), 351-366. DOI: 10.1007/s11948-009-9135-4

Callon, M., Lascoumes P. and Barthe Y. (2009). Acting in an Uncertain World: An Essay on Technical Democracy. Cambridge, MA: The MIT Press.

Costamagna, P. and Larrea, M. (2017). Actores facilitadores del desarrollo territorial. Una aproximación desde la construcción social. Bilbao: Publicaciones Deusto. http://www.orkestra.deusto.es/es/investigacion/publicaciones/libros-informes/desarrollo-territorial/1202actores-facilitadores-desarrollo-territorial-construccion-social

Douglas, H. (2009). Science, Policy, and the Value-Free Ideal. Pittsburgh: Pittsburgh University Press.

Eizagirre, A. (2017). Investigación e innovación responsables: retos teóricos y políticos. Sociología. Problemas e Práticas, 83, 99-116. DOI: 10.7458/SPP2017834400

Eizagirre, A., Rodríguez, H. and Ibarra, A. (2017). Politicizing responsible innovation: responsibility as inclusive governance. International Journal of Innovation Studies, 1(1), 20-36. DOI: 10.3724/SP.J.1440.101003

Felt, U. and Wynne, B. (2007). Taking European knowledge society seriously. Report of the Expert Group on Science and Governance to the Science, Economy and Society Directorate, European Commission. https://publications.europa.eu/en/publication-detail/-/publication/5d0e77c7-2948-4ef5-aec7bd18efe3c442/language-en

Greenwood, D. and Levin, M. (2007). Introduction to Action Research (2nd ed.). Thousand Oaks (California): Sage.

Karlsen, J. and Larrea, M. (2015). Desarrollo territorial e investigación acción. Innovación a través del diálogo. Bilbao: Publicaciones Deusto. http://www.orkestra.deusto.es/es/investigacion/publicaciones/librosinformes/desarrollo-territorial/206-desarrollo-territorial-investigacion-accion-innovacion-traves-dialogo

Kuhlmann, S. and Rip, A. (2018). Next-generation innovation policy and grand challenges. Science and Public Policy. DOI: 10.1093/scipol/scy011 
Merton, R. (1942) [1973]. The normative structure of science. In R. Merton and N. Storer (eds.), The Sociology of Science. Theoretical and Empirical Investigations (pp. 267-278, Ch. 13). Chicago: The University of Chicago Press.

Oswald, K., Gaventa, J. and Leach, M. (2017). Introduction: interrogating engaged excellence in research. IDS Bulletin, 47(6), 1-18. DOI: 10.19088/11968-2016.196

Polanyi, M. (1961). The republic of science: its political and economic theory. Minerva, 1(1), 54-74. DOI: 10.1023/A:1026591624255

Rip, A. (2012). Protected spaces of science: their emergence and further evolution in a changing world. In: M. Carrier and G. Michael (eds.), Science in the Context of Application: Methodological Change, Conceptual Transformation, Cultural Reorientation (pp. 197-220, Ch. 14). Dordrecht: Springer.

Tandon, R., Singh, W., Clover, D. and Hall, B. (2017). Knowledge democracy and excellence in engagement. IDS Bulletin, 47(6), 19-36. DOI: 10.19088/1968-2016.197

Trencher, Y., McCormick, K. B. and Doll, C. (2014). Beyond the third mission: exploring the emerging university function of co-creation for sustainability. Science and Public Policy, 41(2), 151-79. DOI: 10.1093/scipol/sct044

Van den Daele, W. (1978). The ambivalent legitimacy of the pursuit of knowledge. In: B. Egbert and G. Michael (eds.), Proceedings of the Conference Science, Society and Education (pp. 23-61, Ch. 2). Amsterdam: Free University Bookshop.

Ziman, J. (1998). Real Science: What It Is, and What It Means. Cambridge: Cambridge University Press. 


\title{
Challenges FOR CO-Generation IN THE SEARCH FOR THE SOCIAL IMPACT OF THE UNIVERSITY: A CASE OF THE Construction of a Dialogic Space Through ACTion RESEARCH
}

\author{
LOS RETOS DE LA CO-GENERACIÓN EN LA BÚSQUEDA DEL IMPACTO SOCIAL DE LA UNIVERSIDAD: \\ UN CASO DE CONSTRUCCIÓN DE UN ESPACIO DIALÓGICO A TRAVÉS DE LA INVESTIGACIÓN ACCIÓN \\ Patricia Canto $^{1 *}$, Pablo Costamagna ${ }^{2}$, Andoni Eizagirre $^{3}$, and Miren Larrea ${ }^{1}$ \\ Orkestra-Basque Institute of Competitiveness, University of Deusto, Spain ${ }^{1}$ \\ *corresponding author: pcanto@orkestra.deusto.es \\ National Technological University, FRRA/National University Rafaela, Argentina ${ }^{2}$ \\ Mondragon University, Spain ${ }^{3}$
}

\begin{abstract}
At the center of the debate about the social impact of the university we find a new division of work in the production of knowledge that can respond to the challenges of society. This new division of work is more complex and signals an epistemological turn in the form of organizing interaction between academic knowledge and society. This article poses four challenges that were identified based on a case of the construction of a dialogic space for the co-generation of knowledge. The case, which was developed through action research, questions the scheme in which knowledge generated in a university is packaged in the form of articles, books, reports, or conferences and communicated linearly, and suggests another that is based on dialogue and that allows other actors to contribute their knowledge as well.
\end{abstract}

Key words: university, social impact, action research, communication.

\section{Introduction}

The debate about the social impact of the university has been gaining strength in recent years. At the center of this debate lies the ability of the university to interact with other actors in society to co-generate knowledge that contributes to dealing with challenges faced by territories. Public administration policies also foster this debate with a clear commitment to transdisciplinary research aimed at the solution of great social challenges.

Nevertheless, in order for the university to be able to co-generate knowledge with other actors in society, it needs to reformulate its mission of transference as a mission of service to society (Tandon et al., 2017). It should also cast a critical eye on the monopoly it has maintained in the production of knowledge and on its structure in disciplinary silos. Likewise, its researchers need to develop specific abilities, such as active listening and communication in multidirectional dialogues in real time (Oswald et al., 2017), conflict management, power relationships, and knowing how to generate social capital (Karlsen \& Larrea, 2015). Communication, language, and mediation also stand out as necessary abilities in order for the university to play the role of agent of change in the territory (Aranguren et al., 2016; Trencher et al., 2014).

The present article takes a close look at this discussion and aims at responding to the question of what challenges the university faces to cogenerate knowledge with other actors in society. Specifically, it questions the scheme in which knowledge generated in the university is packaged in the form of articles, books, reports, or conferences and communicated linearly to other actors in the territory, without any dialogue that

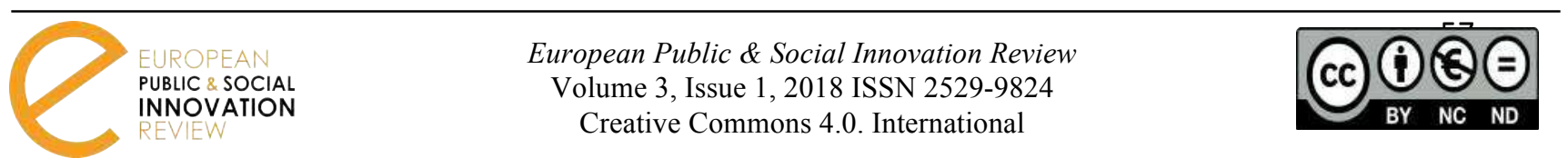


would permit those actors to contribute their own knowledge.

This article is divided into three sections. The first section examines the relationship between the university and society, and brings to light the need to explore a new division of work in the production of knowledge that would be able to respond to the challenges of society. The second section presents the case under consideration: first, the context in which the case develops is established; next, the point of departure is explained - the draft of a book that was initially going to be communicated under the linear scheme described above; and finally, the section concludes with a description of the process of construction of a dialogic space in which to communicate that knowledge through action research. The last section of the article discusses the case by way of conclusion.

\section{The university and its relationship with society}

The idea of research in the university has traditionally been supported by a demarcationist and linear approach to the scientific and social aspects of the research, maintained in the name of objectivity when facing problems. That tradition linked academic research to the search for true, productive, and coherent knowledge, a search governed exclusively by cognitive values and a set of methods through which genuine knowledge is certified. To this characteristic another normative one was added, according to which the guarantee of good science, or of responsible research, is the action of the researcher him- or herself, based on respect for scientific norms (Douglas, 2009). Thus, led by curiosity and his or her desire to discover and interpret the internal evidence of the real, physical, and social world, the researcher would isolate from its rules and norms the consequences of the use of generated knowledge, the satisfaction of human needs, and knowledge that is beneficial for society (Brown \& Guston, 2009).

In this sense, the university and its relationship with society have been governed by a clear functional and moral division of work (Van den Daele, 1978). More specifically, epistemic authority and the organization of research have been subjected to a set of assumptions that guide and make sense of the production of knowledge.
The classical sociological perspective holds that "the institutional goal of science is the extension of certified knowledge" (Merton, 1942: 270). This characteristic of science is observed in a principle that recognizes and encourages the task of the researcher, that is, the freedom and independence of academic research with the goal of generating knowledge for its own sake. Together with this, standardized and professional recognition is established according to the degree of authenticity, scientific value, originality, and the publicizing of knowledge (Polanyi, 1961). All of this is preceded by a linear and positivist view of research that has been of benefit to a model of conduct and evaluation characterized by a quantitative methodology, a cumulative model of scientific knowledge, and a logic with universal pretensions

This image of research and its relationship with society, never exempt from debate, is subject to continual revision (Rip, 2012). In that regard, there are very diverse beliefs: some insist on the complex nature of the challenges that must be addressed as a society, others note the economic and social contribution of the university, and still others focus on the increase in cognitive capital with respect to organizations outside the academic and university network, or emphasize a different image of the expected results. In any case, these changes, although of different forms and degrees, alert us to the need to encourage collaborative programs and strategies in order to boost the intensity, quality, and density of research. In other words, these beliefs make it possible to catch a glimpse of an epistemological and social turn in the form of understanding and organizing interactions between academic knowledge and the institutional, community, and corporate life (Bonaccorsi \& Daraio, 2007). All of this leads to a demand for more socially robust linking policies at the same time that the demarcationist approaches mentioned above being revealed as highly ineffective (Felt \& Wynne, 2007). As a result, research policies are gradually placing more emphasis on problems and challenges to resolve, instead of on disciplines and areas of knowledge, while a greater contextual sensibility emphasizes the widespread and collective nature of knowledge (Kuhlmann \& Rip, 2018). In this way, the traditional assumption about expert discovery as a revelation of objective truths is being replaced by a perspective according to which different values, knowledge, and expectations are integrated into research and innovation processes and the results 
align better with different social visions, needs, and preferences (Eizagirre, 2017).

This to some extent calls into question the idea that a special characteristic of research in the university is the belief that knowledge itself is the primary product and purpose of research. Nevertheless, the emergence of a new modality of organizing, producing, and validating knowledge in application contexts, a modality that transcends pre-established roles, is not at odds with scientific quality and excellence (Ziman, 1998). Likewise, a growing interest in the resolution of problems and research oriented toward challenges does not necessarily entail the rejection of the idea that science is an epistemic strategy of considerable value, nor the rejection of its social characteristics.

On the contrary, the gradual recognition of the dispersed nature of expertise and of the collaboration of different organizations, all bearers of knowledge, values, and interests, favors adaptation to distinct contexts, environments, and users, as it shows greater variety, robustness, and contextual sensibility (Callon et al., 2009). These changes indicate a new division of work, one that is more complex and interactive, and that indicates a system of dispersed research in which different professionals and communities of civil society interact and collaborate in response to social needs and aspirations.

This, however, requires a unique perspective about the nature, meaning, and purposes of the university. On the one hand, it subjects epistemic authority and the organization of research to cooperation with other extra-academic actors and proposals. On the other hand, it advocates for understanding the university in more relational terms and defends the open nature of research and innovation processes (Eizagirre et al., 2017).

\section{Etorikizuna Eraikiz as an experimentation space of the co- generative role of the university: a meeting place for facilitators}

\subsection{Introduction to the case}

The preceding section suggests the need to explore a new division of work, one that is more complex and interactive when it comes time to generate knowledge that responds to the problems of society. For this purpose, it was said that it is necessary to have interaction and collaboration among university researchers from different disciplines, and among those researchers and different professionals and groups of civil society.

The case addressed below is a project developed for the purpose of constructing a meeting place for facilitators of territorial development in the context of Etorkizuna Eraikiz (Building the Future), a program led by the Provincial Government of Gipuzkoa (PGG). This project can be interpreted as an experimental process of the construction of a place for collaboration, not only among researchers, but also among researchers and different professionals, in this case associated with the facilitation of territorial development. It is thus an experiment about the interactive role of the university with other actors in the territory. Following the language used in action research, which inspired the methodology of the present process, it is an experiment about the co-generative role of the university in its goal of generating knowledge that is useful in resolving the problems of society.

The co-generative role of the university is presented in the project from the perspective of the communication of academic knowledge, a field that one of the authors of the present article addresses in her doctoral dissertation. The initial proposal was that a university that wishes to cogenerate knowledge to respond more directly to the needs of society cannot continue to support a scheme of linear communication with society. In such a scheme, knowledge is generated in the university and is then communicated to actors in formats such as reports, articles, books, and seminars without the actors of the territory having the option to dialogue with the researchers or contribute to the construction of the knowledge in question. Two of the authors of the present article were then challenged to experience a different way of communicating a draft of a book that they were working on. The contents had been constructed for book format, and had therefore followed the regular process of academic production. Nevertheless, the work done on the draft makes it possible to speak about a new way of communicating academic knowledge that moves forward in the direction suggested in the theoretical framework by breaking with the traditional division of work.

\subsection{Point of departure: a book draft}

As stated, the point of departure of the process was a draft written by two of the authors of the present 
article that had been planned to be communicated in the form of a book. The content of the book could, a priori, seem insignificant in terms of reflection about how it was communicated. Nevertheless, in order to understand more clearly who the actors were with whom knowledge was later co-generated, the content of the draft should be understood. Thus, let us now introduce the draft that was later converted into a book in Deusto's Territorial Development Series under the title Actores Facilitadores del Desarrollo Territorial. Una Aproximación desde la Construcción Social (Facilitative Actors of Territorial Development. A Social Construction Approach) (Costamagna \& Larrea, 2017).

The book presents the initial argument that facilitative people are a relevant element for processes of territorial development to work, but that their role has nevertheless been conceptualized very little and when it has been done, a neutral role has been attributed to them that does not correspond to the authors' interpretation of their nature in practice. Thus, the book suggests steps to take in order to reinterpret the figure of the facilitator and to help the reader to become a facilitative person of processes of territorial development.

The first step is to understand the origin of this interpretation of facilitation, which is double: the pedagogical focus (developed in Latin America) and action research for territorial development (which has its roots in the collaboration between research environments in the Basque Country and Norway). These approaches make it possible to argue that territorial development is a complex process, and complex processes cannot be developed in terms of order and authority, nor do they allow "finding" solutions through analysis. Rather, solutions must be "constructed" and that requires developing processes of social construction. The book maintains that often in territorial development, complicated problems (for which there exists a solution that can be discovered through expert knowledge) are not distinguished from complex ones (problems for which no solution that can be discovered exists, but rather for which a solution must be constructed in a contextual way). Because of this, people have tried to solve complex problems as if they were complicated ones, through linear logic and hierarchical decisions. However, the figure of the facilitative person has been proposed as someone who can generate the appropriate conditions for other actors to reflect, make decisions, and act to construct solutions in a collaborative way.

After outlining this framework, the authors of the book propose the figure of the facilitative actor, which breaks away from the image of the neutral professional facilitator and suggests that the different actors in the territory, whether from a political, business, or university background, can have their own interests and still be legitimized by other actors to facilitate a process. Once this figure has been defined, the book addresses the leadership of the facilitative actor, which is defined as a relational leadership. The book also poses the question of whether facilitators are born or made and, therefore, whether it is possible to train facilitators. Beginning from the hypothesis that it is, a set of roles of facilitative people are suggested, as are the individual and group abilities needed for those roles to be addressed.

The authors' goal is to invite the reader to reflect about his or her own role in territorial development by seeing whether the conceptual framework of facilitation and its characteristics are helpful to understand some of his or her experiences.

This content was appropriate for participants to experience a new way of communicating academic knowledge, not only because the authors of the book were willing to participate in the process, but also because the content itself is oriented toward generating facilitative abilities in a context of multidisciplinarity and co-generation. Thus, it was foreseeable that the potential readers of the book would be people with an attitude that was a priori favorable toward participating in a cogenerative process.

Considering the direct participation of all the authors of the present article in the action research process that constructed the dialogic space, we have chosen to use the third person plural in the following sections.

\subsection{The construction, through action research, of a dialogic space in which to communicate the book about facilitators}

In order to answer the question of "how" to communicate the book about facilitative actors, we developed an action research process based on the co-generative model of Greenwood and Levin (2007). Action research is proposed as a strategy for change that, through cycles of reflectionaction-reflection, generates lessons that solve a 
problem in practice and generate new knowledge that is relevant in the academic environment.

As mentioned above, all four authors of the present article participated in the action research process. We began with our goal, which was defined in terms of "communicating the book in a co-generative way," but we played different roles. The authors of the book played the role of owners of the problem, while the author who was writing her doctoral dissertation at the time designed the process based on an analytical framework constructed on the basis of a review of the literature on Responsible Research and Innovation (RRI) and Communication for Development and Social Change (CDSC). For his part, the fourth author, who played the role of critical friend, maintained constant dialogue with the doctoral student, helping her to reflect on the development of the process and suggesting new lines of discussion. At different times, professionals with knowledge about information technologies, audiovisual communication and design, and project management were integrated. In the final stage a pilot group formed primarily by doctoral students and researchers also participated.

In each of the workshops, the conceptual frameworks presented by the doctoral student shed light on the book authors' reflections on the challenge expressed as "how" to communicate knowledge about the figure of the facilitative actor. Based on those reflections, the authors made decisions that were put into action. By the time the process was finalized, those actions had materialized in a digital dialogic space in order to communicate knowledge about the figure of the facilitative actor. Table 1 shows a summary of the process.

Table 1. The action research process: concepts and decisions.

\begin{tabular}{|l|l|}
\hline $\begin{array}{l}\text { Concept/ } \\
\text { Date of workshop }\end{array}$ & \multicolumn{1}{c|}{ Decisions } \\
\hline $\begin{array}{l}\text { Communication as a } \\
\text { process. } \\
\text { (March 7, 2017) }\end{array}$ & $\begin{array}{l}\text { Design a journey with two stages: } \\
\text { - An interactive journey on which travelers internalize the contributions of } \\
\text { the authors and of travelers who have already visited the different stops. } \\
\text { - A meeting place at the end of the trail in which the book will continue } \\
\text { being written. }\end{array}$ \\
\hline $\begin{array}{l}\text { The interaction of } \\
\text { different knowledge } \\
\text { sets. } \\
\text { (April 12, 2017) }\end{array}$ & $\begin{array}{l}\text { - Design the interactive journey with spaces that make it possible to } \\
\text { visualize the idea that at each stop on the journey a deferred dialogue takes } \\
\text { place between the authors and the travelers about the different chapters of } \\
\text { the book. }\end{array}$ \\
\hline $\begin{array}{l}\text { The inclusion of } \\
\text { perspectives, } \\
\text { experiences, and } \\
\text { expectations. } \\
\text { (May 9, 2017) }\end{array}$ & $\begin{array}{l}\text { - Formulate questions at each stop that invite the travelers to reflect about } \\
\text { the chapters of the book by connecting with their own theoretical and } \\
\text { practical knowledge. }\end{array}$ \\
\hline $\begin{array}{l}\text { Learning } \\
\text { communities. } \\
\text { (June 13, 2017) }\end{array}$ & $\begin{array}{l}\text { - That the meeting place at the end of the trail should be constructed based } \\
\text { on an emerging dialogue among the travelers who have completed the } \\
\text { journey. } \\
\text { - That the responsibility for keeping the dialogue alive should be shared. }\end{array}$ \\
\hline
\end{tabular}

Source: present study.

\subsection{Result: a meeting place for facilitative actors}

The tangible result of the process described in the previous section is an open space online (https://dgroups.org/groups/perfadt) in which participants are invited to carry out a journey that ends in an agora or plaza where they can meet with 
other travelers interested in the facilitation of territorial development.

The need to design a realistic and sustainable dialogic process was a matter of constant concern throughout the workshops. This reflection was key in the design of the space as a process divided into the two stages mentioned above: the journey and the plaza.

\section{a. The interactive journey}

The interactive journey is set up as a deferred dialogue that is developed throughout a tour with different stops, at each of which the travelers have the possibility of accessing both the original chapters written by the authors and audiovisual materials prepared by the authors in order to make those chapters more accessible to the travelers. But above all, the travelers have the possibility of sharing their reflections about the contents of the book and reading the contributions of travelers who reached the stops earlier. Following the criteria of realism and sustainability, this deferred dialogue is an intermediate method between the total absence of dialogue and dialogue in real time among the authors and readers of the book. This intermediate method was considered necessary because the traditional format of communication of knowledge through a book would not have allowed for the development of any type of dialogue, while the need to maintain a continual dialogue would have been unsustainable for the researchers. The concept of deferred dialogue made it possible to find an intermediate point between the two extremes.

Figure 1 shows one of the stops on the journey. The space on the left consists of the contributions of the authors of the book expressed through texts and audiovisual materials; videos in which the authors directly address the travelers complement the chapters. The space on the right consists of the travelers' contributions.

Figure 1. An interactive journey in which a deferred dialogue is developed.

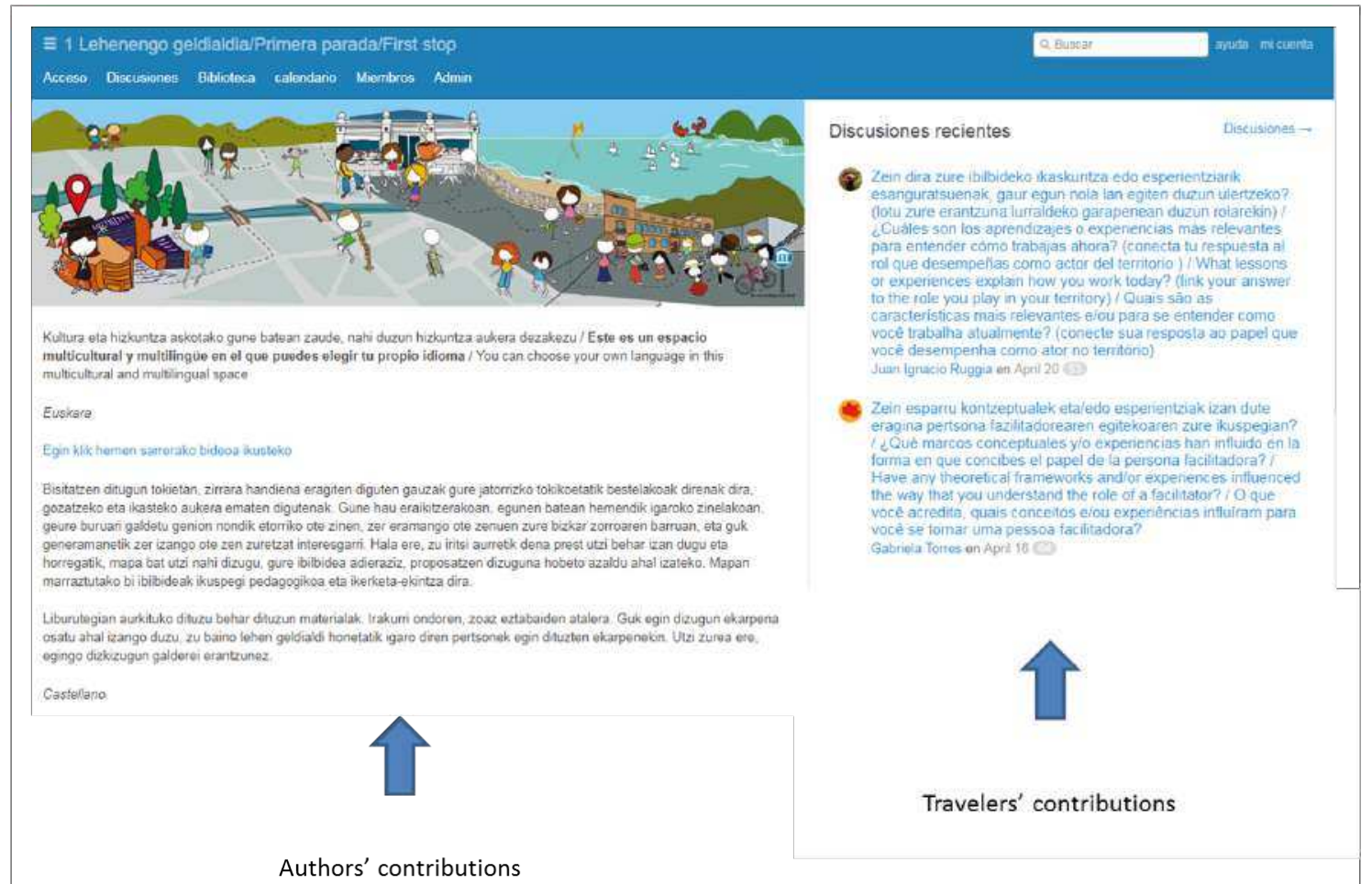

Source: https://dgroups.org/groups/perfadt 
At the time of writing, a total of 183 people from different parts of the world have begun the journey: Argentina, 73; Basque Country, 51; Uruguay, 22; Brazil, 9; Mexico, 6; Finland, 5; Colombia, 3; Peru, 3; and 1 person each from Germany, Chile, Cuba, Ecuador, Italy, Kenya, Nigeria, Norway, United Kingdom, the Dominican Republic and Vietnam. Among them are lecturers, researchers, and makers of public and private policy. b. The agora or plaza as a meeting point

The second stage of the process, which is less closely linked to the direct contents of the book and is more directly interactive, was defined as a space in which to "continue writing the book." It was configured as an agora, an emerging space that requires the more constant involvement of the authors but in which the responsibility for keeping the dialogue alive is shared. That is, the book continues to be written, but no longer by the authors alone but by all the people who make up the space and bring up debates and reflections about the facilitation of territorial development. Figure 2 shows this emerging space.

Figure 2. An emerging space in which to continue writing the book.

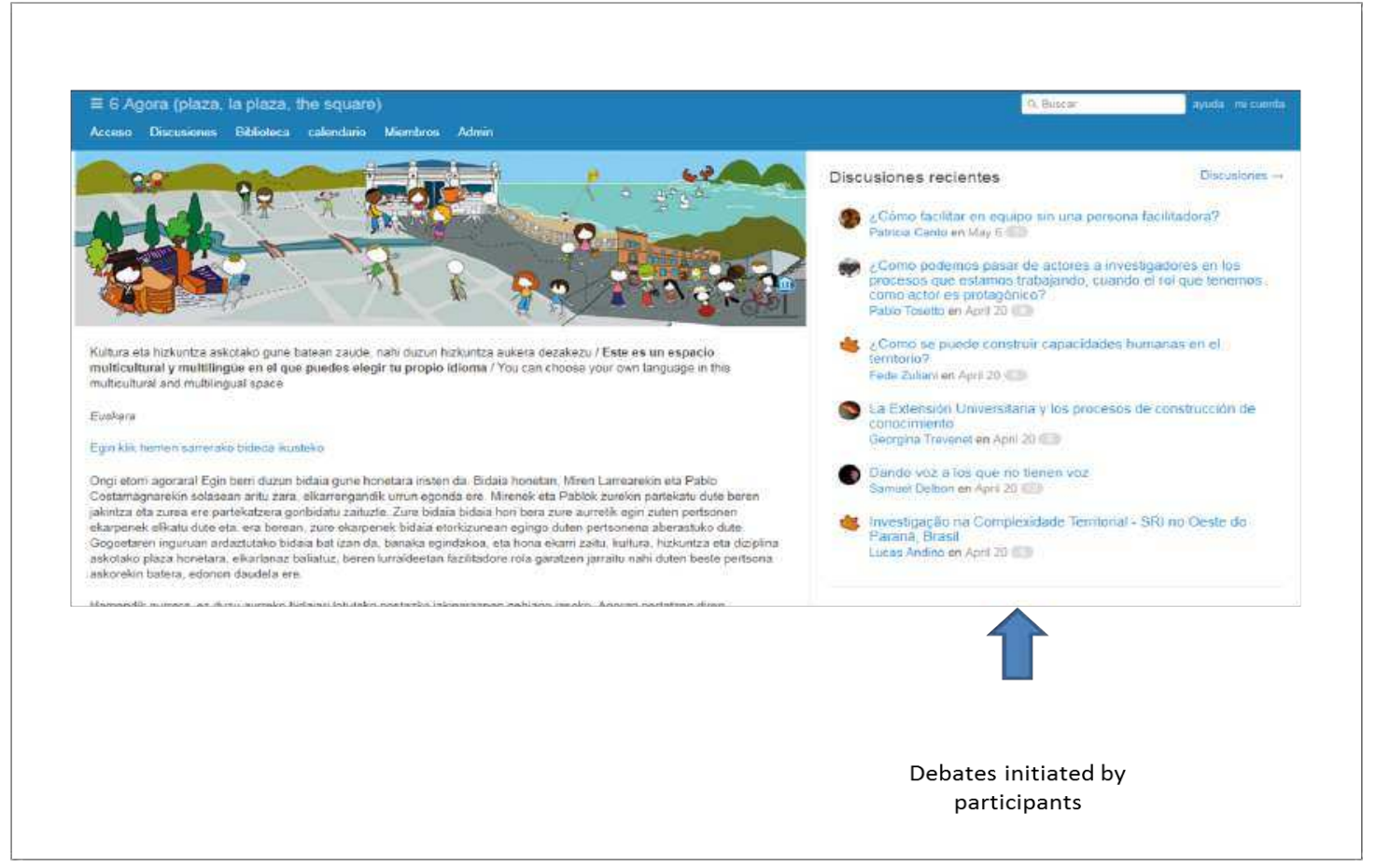

Source: https://dgroups.org/groups/perfadt

At the time of writing, of the 183 people who started the journey, 54 have integrated themselves into the agora and 15 have participated in the debates. The 54 people are distributed as follows: Argentina, 31; Basque Country, 10; Uruguay, 6; Finland, 3; and 1 person each from Brazil, Italy, Mexico, and Nigeria.
The following is an example of one of the discussions that evolved in the agora, which connects with the discussion about the invisibility of the facilitative person brought up in the book: ${ }^{1}$

\footnotetext{
${ }^{1}$ Permission for publication in the present article has been granted by the authors of these extracts.
} 
"I would like to share a very recent experience I had in an action research project that I facilitate. At a certain point, I realized that I was becoming invisible as a facilitator. It happened when one of the policy-makers of the project, in a presentation to introduce her strategy to support local development, referred to the actions defined as a result of the action research process but did not mention the reflection-action space that she shares with us, the researchers $[\ldots]$ "

Facilitators' Space, November 18, 2017

The discussion that followed that intervention included the following extract:

"I think that part of the reason why the role of facilitators in processes of territorial development is not valued is due to the fact that many of us who acted as facilitative people believed in the importance of the invisibility of that role. Now I believe the opposite."

Facilitators' Space, November 21, 2017

This debate generated a total of 24 interventions in which different actors in the territory (researchers and others) engaged in a dialogue about this issue that is addressed in the book but that in the dialogue is reformulated based on the experiences of the different actors.

We understand that with these processes, a process of co-generation comes into being, cogeneration of knowledge that is associated with the book, but goes beyond the contents presented in the book. For this reason, our interpretation is that this means of communicating the book is a response to the new co-generative modes that are proposed as a challenge to the university and that can open pathways to processes of construction of knowledge that are co-generative and aimed at the resolution of specific problems. The division of work is modified, and there is a group perspective in which the knowledge of the actors is valued.

One of the characteristics of this process is that contents created by the researchers were present from the beginning. In this approach therefore, the researchers' initial conceptualization and theorization efforts are not substituted by a space in which knowledge is constructed dialogically. What is proposed is the complementarity of both approaches in a framework in which there are times when the researchers work on their own and times when they interact with their "readers" in new knowledge construction processes.

\section{Discussion and conclusions}

Having shared the theoretical framework and a practical case, let us now specify the connections between them in order to be able to construct the conclusions of the article. Considering that we stand before a debate that is gaining strength, but for which the university does not always have answers, we present the discussion not in terms of recommendations, but as centering around four challenges:

a) To create socially relevant knowledge;

b) To overcome the division of functions in the creation of knowledge: the university creates it and society assimilates it;

c) The legitimization of co-generated knowledge; and

d) A change of culture within the university in order to be able to co-generate.

The first challenge for the university that we identified in the first section is that of generating socially relevant knowledge. For this purpose it is necessary to go beyond epistemological, methodological, or theoretical approaches that organize the production of knowledge around disciplinary silos since the complexity of the challenges of society requires views shaped by interaction among different disciplines.

Multidisciplinarity has entered the project in two different ways. First, the design of the interactive communication space for the book about facilitative actors is the result of a multidisciplinary view (economy, communication, philosophy, information technologies, and audiovisual design) of a specific problem: the need for the university to contribute its knowledge in a way that contributes to change in the territory. On the other hand, the space is designed for experimentation with interaction among different disciplines and among different academic and extra-academic actors.

One of the people who completed the journey shared that what he liked best was the following: 
"...being able to learn about the experiences and opinions of other actors. Above all, my own internal debate when I disagreed with what was being presented or understood it in a new way, and being able to work on it in order to construct a joint vision."

Quote from the questionnaire filled out by travelers who completed their interactive journey (January 11, 2018).

The second challenge is that it is not enough to escape the logic of disciplinary silos, it is also necessary to go beyond the division of functions, for which reason it often goes without saying that the university produces knowledge and then society receives it and applies it. The interactive strategy for the communication of knowledge about facilitative actors attempts to overcome that division by creating a space in which "the book continues to be written" but in which the responsibility for writing it no longer belongs solely to the authors but to all who define that space. This transition to new forms of knowledge production includes the difficult task of combining the criteria of scientific quality with social relevance and contextual sensibility.

This requires a change in the university, given that areas endowed with knowledge are extended and decentralized, so that it should be able to deploy new abilities associated with mutual recognition, collaboration, and cooperative learning. But not only the university is faced with the need to change. This type of process also demands change in other areas of society in which the territorial actors must make the transition from passive receptors to co-generators of knowledge, with the attendant fears, lack of practice, and time devoted to reflection. One of the participants in the facilitators' space expressed this idea as follows:

"The effort is in understanding that it is a space for group learning, where all opinions generate contributions... easy to say, but a bit more complicated to carry out...

Facilitators' Space, April 6, 2018

The above leads us to the third challenge, which was posed on the theoretical level in the first section: how to legitimize in academia the new knowledge co-generated between science and society? The co-generative character improves the chances for the generated knowledge to help resolve problems when the knowledge of the actors who are most familiar with those problems is integrated in the process. But as it is not a knowledge produced under the supposed imperatives regarding scientific production, the cogenerated knowledge poses difficulties when it comes time to integrate it again in the academic debate. It is for this reason that it is important to construct mechanisms of legitimization of this type of knowledge in academia, so that a collaborative and open type of generation of knowledge does not entail penalization in researchers' careers.

As an example, research that incorporates different perspectives in its design, includes different actors, diversifies its sources of knowledge, reflects actively on ways to integrate socio-economic considerations, and is flexible enough to change direction and orientation in response to lessons, values and needs, such research appeals to a profound revision of the traditional epistemological and methodological suppositions about the nature and sense of research. To this are added other obstacles, such as those referring to the times and purposes of investigative activity, to the organizational and functional structure of universities, and to the models and criteria that regulate and incentivize the different programs for quality evaluation and accreditation. Regarding the latter, evaluative structures for national accreditation that regulate the system of professional promotion and its institutions and that control and incentivize research, publication, and academic careers in universities are clearly intended for a very singular model of university that creates difficulties in integrating proposals like that presented in this article. The question remains: How do we legitimize knowledge co-generated in the register proposed here?

The fourth and final challenge, which is related to the preceding three, is the change of culture required in the university in order to be able to naturally integrate co-generative models. How does the university today deal with the call for co-generation? How are individual researchers positioned with respect to the facilitation of processes of co-generation of knowledge among different actors? This requires a profound change in routines and behaviors that goes beyond any individual decision by the researcher. Ultimately, it is a matter of rethinking the aspects that affect the 
process and purpose of the research. Some of the difficulties and resistances were mentioned above, many of them of an organizational or structural nature. In any case, we must also create the foundations for a cultural change within the university and in the short term, in the specific context analyzed in the present article. Such a change could come about thanks to doctoral courses and training for researchers in which these types of processes are presented. The constructed facilitators' meeting place could serve this purpose.

In this sense, we believe it necessary to highlight the importance of experimentation. Thanks to the Etorkizuna Eraikiz public administration program, the authors of the present article have had the opportunity to experiment with a space in which academic knowledge is shared dialogically. This opportunity has created the conditions necessary to generate abilities in the university itself and in other actors in the territory within the framework of the unique governance possible on this journey, a governance in which the different actors, researchers, and non-researchers collaborate in the construction of knowledge that helps to overcome the challenges of the territory.

\section{Acknowledgements}

The authors would like to thank Rakel Vázquez, Maite Reizabal, and Idoia Egaña for contributing their knowledge in the construction of the dialogic space. They would also like to thank the Provincial Council of Gipuzkoa for support in the development of the project "University and Collaborative Governance: Deploying Facilitation Capabilities".

\section{References}

Aranguren, M. J., Franco, S., Horta, R. and Silveira, L. (2016). Retos y aprendizajes de institutos de investigación transformadora. Journal of Technology Management and Innovation, 11(1), 69-79. DOI: 10.4067/S071827242016000100010

Bonaccorsi, A. and Daraio, C. (2007). Universities as Strategic Knowledge Creation. Specialization and Performance in Europe. Cheltenham: Edward Elgar Publishing. DOI: 10.4337/9781847206848

Brown, M. and Guston, D. (2009). Science, democracy, and the right to research. Science and Engineering Ethics, 15(3), 351-366. DOI: 10.1007/s11948-009-9135-4

Callon, M., Lascoumes P. and Barthe Y. (2009). Acting in an Uncertain World: An Essay on Technical Democracy. Cambridge, MA: The MIT Press.

Costamagna, P. and Larrea, M. (2017). Actores facilitadores del desarrollo territorial. Una aproximación desde la construcción social. Bilbao: Publicaciones Deusto. http://www.orkestra.deusto.es/es/investigacion/publicaciones/libros-informes/desarrollo-territorial/1202actores-facilitadores-desarrollo-territorial-construccion-social

Douglas, H. (2009). Science, Policy, and the Value-Free Ideal. Pittsburgh: Pittsburgh University Press.

Eizagirre, A. (2017). Investigación e innovación responsables: retos teóricos y políticos. Sociología. Problemas e Práticas, 83, 99-116. DOI: 10.7458/SPP2017834400

Eizagirre, A., Rodríguez, H. and Ibarra, A. (2017). Politicizing responsible innovation: responsibility as inclusive governance. International Journal of Innovation Studies, 1(1), 20-36. DOI: 10.3724/SP.J.1440.101003

Felt, U. and Wynne, B. (2007). Taking European knowledge society seriously. Report of the Expert Group on Science and Governance to the Science, Economy and Society Directorate, European Commission. https://publications.europa.eu/en/publication-detail/-/publication/5d0e77c7-2948-4ef5-aec7bd18efe3c442/language-en

Greenwood, D. and Levin, M. (2007). Introduction to Action Research (2nd ed.). Thousand Oaks (California): Sage.

Karlsen, J. and Larrea, M. (2015). Desarrollo territorial e investigación acción. Innovación a través del diálogo. Bilbao: Publicaciones Deusto. http://www.orkestra.deusto.es/es/investigacion/publicaciones/librosinformes/desarrollo-territorial/206-desarrollo-territorial-investigacion-accion-innovacion-traves-dialogo

Kuhlmann, S. and Rip, A. (2018). Next-generation innovation policy and grand challenges. Science and Public Policy. DOI: 10.1093/scipol/scy011

Merton, R. (1942) [1973]. The normative structure of science. In R. Merton and N. Storer (eds.), The Sociology of Science. Theoretical and Empirical Investigations (pp. 267-278, Ch. 13). Chicago: The University of Chicago Press. 
Oswald, K., Gaventa, J. and Leach, M. (2017). Introduction: interrogating engaged excellence in research. IDS Bulletin, 47(6), 1-18. DOI: 10.19088/11968-2016.196

Polanyi, M. (1961). The republic of science: its political and economic theory. Minerva, 1(1), 54-74. DOI: 10.1023/A:1026591624255

Rip, A. (2012). Protected spaces of science: their emergence and further evolution in a changing world. In: M. Carrier and G. Michael (eds.), Science in the Context of Application: Methodological Change, Conceptual Transformation, Cultural Reorientation (pp. 197-220, Ch. 14). Dordrecht: Springer.

Tandon, R., Singh, W., Clover, D. and Hall, B. (2017). Knowledge democracy and excellence in engagement. IDS Bulletin, 47(6), 19-36. DOI: 10.19088/1968-2016.197

Trencher, Y., McCormick, K. B. and Doll, C. (2014). Beyond the third mission: exploring the emerging university function of co-creation for sustainability. Science and Public Policy, 41(2), 151-79. DOI: 10.1093/scipol/sct044

Van den Daele, W. (1978). The ambivalent legitimacy of the pursuit of knowledge. In: B. Egbert and G. Michael (eds.), Proceedings of the Conference Science, Society and Education (pp. 23-61, Ch. 2). Amsterdam: Free University Bookshop.

Ziman, J. (1998). Real Science: What It Is, and What It Means. Cambridge: Cambridge University Press. 